\title{
Ciprofloxacin does not Prolong the QTc Interval: A Clinical Study in ICU Patients and Review of the Literature
}

Charlotte PM Heemskerk ${ }^{\text {a,b }}$, Evelien Woldman ${ }^{\text {a,c }}$, Marieke Pereboom ${ }^{\text {a }}$, Ruud TM van der Hoeven ${ }^{\text {a }}$, Aukje K MantelTeeuwisse $^{\mathrm{b}}$, Claudia W van Gemeren ${ }^{\mathrm{d}}$, Matthijs L Becker ${ }^{\mathrm{a}}$

aPharmacy Foundation of Haarlem Hospitals, Haarlem, the Netherlands; ${ }^{b}$ Division of Pharmacoepidemiology \& Clinical Pharmacology, Utrecht Institute for Pharmaceutical Sciences (UIPS), Utrecht, the Netherlands; ${ }^{\mathrm{c}}$ Meander Medical Center,

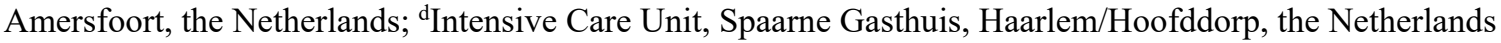

Received June 3, 2017; Revised, September 5, 2017; Accepted, September 10, 2017, October 10, 2017.

Summary - Purpose. Ciprofloxacin may prolong the QT interval and increase the risk of Torsade de Pointes (TdP). Intravenous administration of ciprofloxacin in patients with additional risks may elevate the risk of QTc interval prolongation. We prospectively assessed whether intravenous ciprofloxacin prolongs the QT interval in patients with additional co-morbidities and risk factors. We also reviewed the literature on the QT prolonging effect or TdP inducing effect of ciprofloxacin. Methods. ICU Patients who were treated with intravenous ciprofloxacin as part of their therapy were recruited. ECG was recorded within 60 min before start and in the last $30 \mathrm{~min}$ of $1 \mathrm{~h}$ infusion, or within $30 \mathrm{~min}$ after the end of ciprofloxacin infusion. QT interval was corrected for the heart rate using both Bazett's and Fridericia's formula. The changes were analyzed using the paired Student's t-test. Results. Ten patients were included in the study (average age 74-y, 6 males). The average baseline QTc interval corrected with Bazett's formula was $448 \mathrm{~ms}$ that was shortened during or after ciprofloxacin infusion by $3 \mathrm{~ms}$ and $2 \mathrm{~ms}$ based on Bazett's $(p=0.67)$ and Fridericia's $(p=0.68)$ formula, respectively. No observational study or cohort study thus far has shown that ciprofloxacin has a QT prolonging effect or increases the risk of TdP or (cardiovascular) mortality. Conclusion. Based on our results and the results of previous studies, it is unlikely that ciprofloxacin has a clinically relevant QT prolonging effect or an increased risk of TdP.

This article is open to POST-PUBLICATION REVIEW. Registered readers (see "For Readers") may comment by clicking on ABSTRACT on the issue's contents page.

\section{INTRODUCTION}

Ciprofloxacin, a fluoroquinolone, is a commonly used antimicrobial agent. Fluoroquinolones are well tolerated; however they are associated with QT interval prolongation on the ECG (1). It is assumed that an increase in QT interval indicates an increased risk of Torsades de Pointes (TdP), a rare but potentially fatal, polymorphic ventricular arrhythmia (2). Grepafloxacin and sparfloxacin, other member of the fluoroquinolones, have been withdrawn worldwide from the market for this reason.

Although QT prolongation is believed to be a class effect of fluoroquinolones differences exist between the various drugs in this group (3). Ciprofloxacin seems to be the fluoroquinolone with the lowest risk of QT prolongation and it is questionable whether ciprofloxacin has a clinically relevant QT prolonging effect $(4,5)$. Ciprofloxacin has been associated with $\mathrm{TdP}$ in various case reports $(5,6)$, but other studies suggested that there is no increased risk of QT prolongation or TdP (712).

All studies thus far have been performed with orally administered ciprofloxacin in healthy volunteers or outpatients. However, ciprofloxacin is frequently given intravenous to inpatients, for example on intensive care units (ICU). Intravenous administration of ciprofloxacin may result in higher plasma concentrations than oral administration and thus result in a higher risk of QT prolongation and TdP. ICU patients may also be more vulnerable to QT prolongation, due to co-morbidity and additional risk factors, such as electrolyte disturbances.

Corresponding Author: Pharmacy Foundation of Haarlem Hospitals, M. L. Becker (Matthijs), Boerhaavelaan 24, 2035 RC Haarlem, The Netherlands; E-mail: mbecker@sahz.nl 
The objective of this study was to determine the change in QTc interval during or directly after administration of intravenous ciprofloxacin in ICU patients.

\section{METHODS}

\section{Study population}

This study was performed in the ICU of the Spaarne Gasthuis (Haarlem/ Hoofddorp, the Netherlands), a general teaching hospital with around 900 beds, between April and July 2016. Patients who were admitted at the ICU and treated with intravenous ciprofloxacin as part of their regular care, were eligible for inclusion. Exclusion criteria were age below 18 years, non sinus rhythm, acute cardiac diseases, deviations in the ECG that disturb the QT interval and use of QT prolonging drugs with known risk of TdP, other than ciprofloxacin, according to the AZCERT list (13). Treatment with haloperidol in a dose of less than $5 \mathrm{mg}$ per day was allowed (14).

\section{Study procedure}

If eligible, the patient or his / her legal representative was asked for informed consent. Two ECGs were taken around one ciprofloxacin administration. The first ECG was taken within the 60 minutes before start of the ciprofloxacin administration. The second ECG was taken in the last 30 minutes of the infusion or within 30 minutes after the end of the infusion. Ciprofloxacin is administered through a 60 minutes intravenous infusion. All ECGs were standard 12-lead resting ECGs $(25 \mathrm{~mm} / \mathrm{s}$ paper speed, $10 \mathrm{~mm} / \mathrm{mV}$ amplitude, and $40 \mathrm{~Hz}$ sampling rate). ECGs were recorded using Hamilton MAC 5500 HD with automated analysis by the MUSE Cardiology Information System. The QT and RR interval as measured by the MUSE information system were used. Both the Bazett's formula $\left(\mathrm{QTc}=\mathrm{QT} / \mathrm{RR}^{1 / 2}\right)$ (15) and the Fridericia formula $\left(\mathrm{QTc}=\mathrm{QT} / \mathrm{RR}^{1 / 3}\right)$ (16) were used to correct for the heart rate.(17)

\section{STATISTICAL ANALYSIS}

Paired t-test was used to analyze statistical significance between the QTc interval before administration and the QTc interval during or shortly after ciprofloxacin infusion. Analyses were performed using SPSS software (version 20.0.0; SPSS, Chicago, IL).
Ethics

The Medical Research Ethics Committee NoordHolland (Alkmaar, the Netherlands) judged that this study does not fall under the scope of the Medical Research Involving Human Subjects Act. Therefore, approval from an Ethics Committee was not needed.

\section{RESULTS}

We included ten patients who signed informed consent and in whom ECGs were taken both before and during or shortly after ciprofloxacin infusion. The average age of the study population was 74 (SD 7,8 ; range 60-85) years and 6 patients were men. In three patients, a reduced dose was given due to renal dysfunction (two patients $400 \mathrm{mg} \mathrm{qd}$, one patient $200 \mathrm{mg}$ bid). All other patients received a dose of $400 \mathrm{mg}$ bid. Seven patients had one or more additional risk factors for QT prolongation beside age and gender. These risk factors were haloperidol use $<5 \mathrm{mg}$ /day $(\mathrm{n}=2)$, recent past use of amiodaron $(\mathrm{n}=1)$, hypokalemia $(\mathrm{n}=1)$, diabetes mellitus $(\mathrm{n}=4)$ and decompensated heart failure $(\mathrm{n}=2)$.

The average QTc interval did not change significantly during or shortly after ciprofloxacin administration versus before administration (table 1 , figure 1). The average QTc interval was $3 \mathrm{~ms}$ shorter calculated with the Bazett's formula $(\mathrm{p}=0.67)$ or $2 \mathrm{~ms}$ shorter if the Fridericia's formula was used $(\mathrm{p}=0.68)$.

\section{DISCUSSION}

In our study, we did not find a QT prolonging effect of ciprofloxacin in ICU patients. This is in line with previous studies in other populations. Makaryus et al. performed an observational study in eleven patients taking oral ciprofloxacin, and studied the change in QTc interval on the ECG before and 2 to 48 hours after the first dose of ciprofloxacin (7). A non-significant shortening of the QTc interval of 10 $\mathrm{ms}$ after administration of ciprofloxacin was found. Noel et al studied the effect on the QTc interval after single-dose oral administration of $1500 \mathrm{mg}$ ciprofloxacin in 48 healthy volunteers (8). This is a supratherapeutic dose, because the maximum oral dose is $750 \mathrm{mg}$ twice a day. They found no statistically significant differences in QTc interval after administration of ciprofloxacin compared with placebo at various time points until 24 hours after administration, although in analyses of the average 
post treatment QTc a minor increase between 2 and $5 \mathrm{~ms}$ was found. Tsikouris did not find a QT prolonging effect after two hours or seven days treatment with orally administered ciprofloxacin in

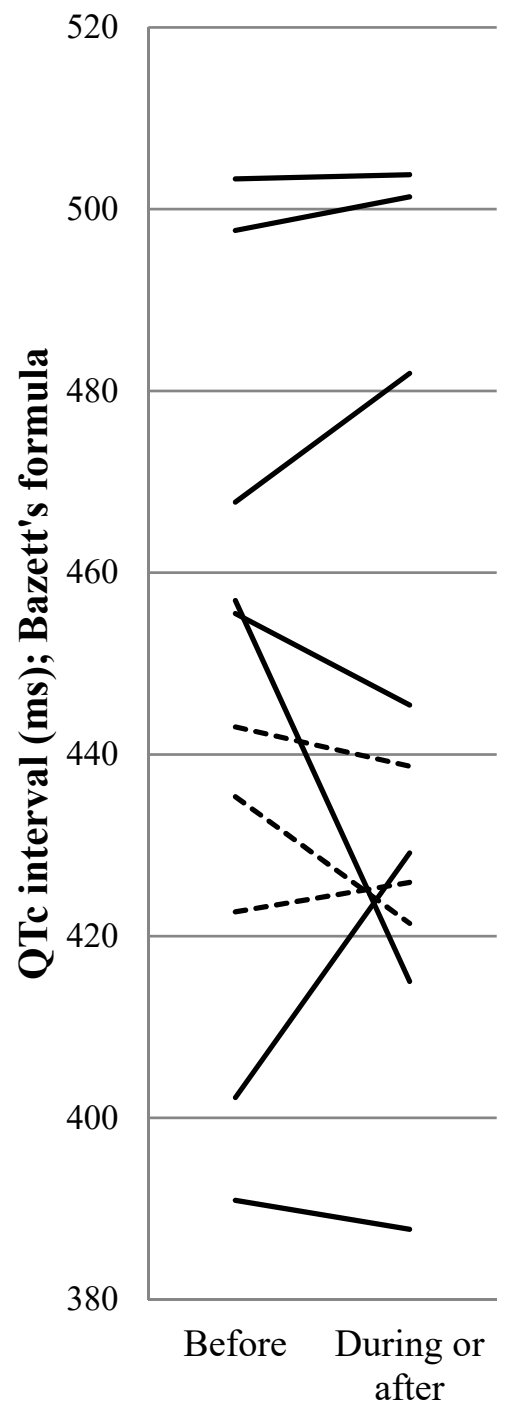

13 healthy volunteers (9). In this study, the QTc interval was prolonged during treatment with moxifloxacin."

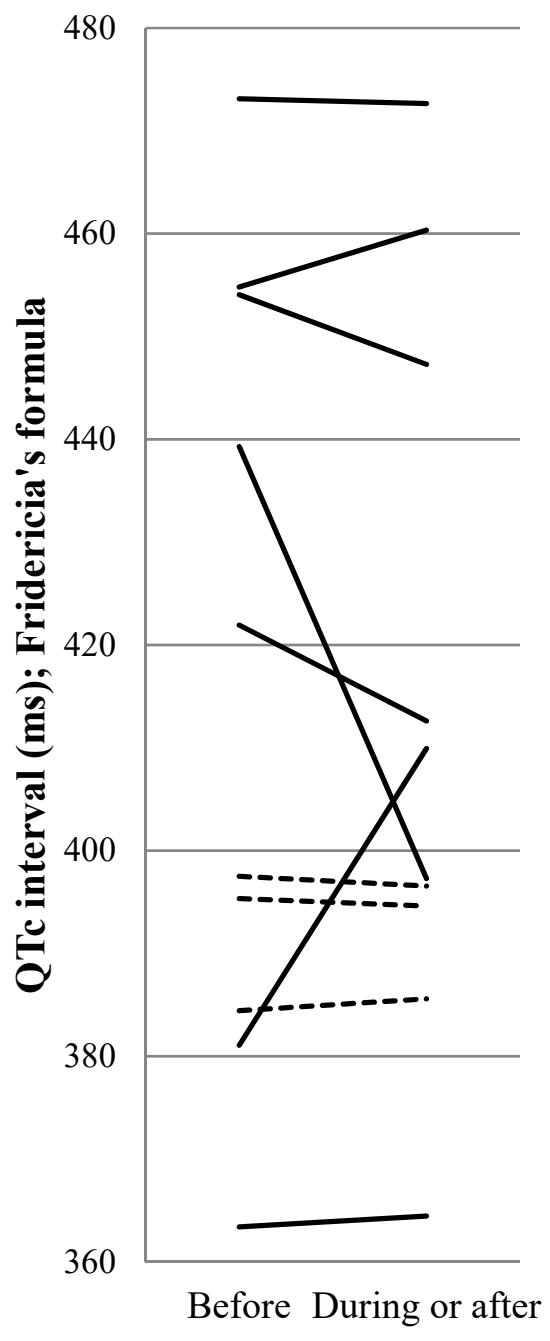

Figure 1. Change in QTc interval during or shortly after intravenous administration of ciprofloxacin for the individual patients, adjusted with the Bazett's formula (left) and Fridericia's formula (right). Patients with risk factors are shown with solid lines.

Table 1. Change in QTc interval during or shortly after intravenous administration of ciprofloxacin

\begin{tabular}{lll}
\hline & Before infusion & During or after infusion \\
\hline Average ventricular rate (SD) & $94(14) \mathrm{bpm}$ & $93(15) \mathrm{bpm}$ \\
\hline Average QTc (SD) - Bazett & $448(37) \mathrm{ms}$ & $445(39) \mathrm{ms}$ \\
Change in QTc, p-value - Bazett & - & $-3 \mathrm{~ms}(\mathrm{p}=0.67)$ \\
Patients with prolonged QTc interval - Bazett ${ }^{\mathrm{a}}$ & 4 & 3 \\
\hline Average QTc (SD) - Fridericia & $416(37) \mathrm{ms}$ & $414(35) \mathrm{ms}$ \\
Change in QTc - Fridericia & - & $-2 \mathrm{~ms}(\mathrm{p}=0.68)$ \\
Patients with prolonged QTc interval - Fridericia & 2 & 1
\end{tabular}

${ }^{a}$ QTc interval above $450 \mathrm{~ms}$ for man or above $470 \mathrm{~ms}$ for woman

$\mathrm{SD}=$ standard deviation 
Three population-based cohort studies have been performed. In a population based cohort study with 265,000 ciprofloxacin prescriptions, no differences in cardiovascular mortality or all-cause mortality were seen during a 10-days course of ciprofloxacin compared with amoxicillin, an antimicrobial agent that is not associated with QT prolongation (10). Similarly, no significant differences in cardiovascular mortality were found in 205,205 ciprofloxacin users compared with amoxicillin-clavulanic acid users (11). On the contrary, the QT prolonging antimicrobial agent's azithromycin and moxifloxacin were associated with an increased risk of cardiovascular mortality $(10,11)$. In the third study, $1,830,000$ prescriptions for ciprofloxacin were compared with prescriptions for penicillin $\mathrm{V}$, and the risk of arrhythmia was not statistically significant increased (12).

On the other hand, twelve case-reports have been published in which ciprofloxacin has been associated with $\operatorname{TdP}(5,6)$. In the majority of these cases, other risk factors, such as co-prescribed QT prolonging drugs, pre-existing heart disease and electrolyte imbalances were present.

Our study has some potential strengths and limitations. We were the first to assess the QT prolonging effect in adult ICU patients. These patients are older, have various co-morbidities and one or more acute illnesses, and therefore are most likely more vulnerable to QT prolongation. Even in these patients, we did not find a QT prolonging effect of intravenous administered ciprofloxacin. After inclusion of the ten patients in our study, we performed an interim analysis. As we did not see even a trend towards a QT prolonging effect, we decided to stop the inclusion of new patients. Ideally, ciprofloxacin concentrations would have been measured while taking ECGs, to relate the prolongation of the QTc interval with the plasma concentrations, and compare these values with patients treated with placebo. However, in our ICU population, this was not possible. We analyzed the QTc interval only twice, both before administration of ciprofloxacin and in the last 30 minutes of the infusion or within 30 minutes after the end of the infusion. The latter time window was chosen, because plasma levels are highest and the effect on the QTc interval at maximum. The change in plasma levels before and during or shortly after intravenous administration of ciprofloxacin is dependent on the frequency of administration and the elimination half-life. The elimination half-life of ciprofloxacin is three to five hours and ciprofloxacin is given twice daily.(18) Therefore, the plasma level before administration is five to sixteen times lower than the plasma level during or shortly after administration. In patients with severe renal impairment, the elimination half-life raises to up to twelve hours, but in these patients ciprofloxacin is given once daily and plasma levels will be four times lower before administration.(18) Drug induced QT interval prolongation is caused by an effect on the cardiac ion channels and change of the ion currents, among which the IKr current is an important one.(2) There is a strong correlation between plasma levels and QTc prolongation, because the heart is very well perfused and binding of the QT prolonging drug on the ion channels has an instant effect on the ion currents. If ciprofloxacin did have a QT interval prolonging effect, these variations in plasma levels before and during or shortly after intravenous ciprofloxacin administration would have been large enough to cause variations in QT interval. More frequent ECG measurements would have reduced intraindividual variation in QTc values and improved the power of the study. Moreover, we did not study the change in QTc values at other time points in our clinical study.

In our study, we did not find a QT prolonging effect of intravenous ciprofloxacin. Our results are in line with previous studies that did not find a QT prolonging effect of orally administered ciprofloxacin in the general population or healthy volunteers. The QT prolonging effect is only suggested because other drugs in the class of fluoroquinolones do prolong the QTc interval and the effect has only been described in various case reports. However, in these case reports other risk factors may have caused the TdP and the infection for which ciprofloxacin has been prescribed, may have increased the vulnerability for TdP. No clinical study thus far has shown that ciprofloxacin has a QT prolonging effect or increases the risk of TdP. In March 2015, ciprofloxacin has been added to the AzCERT list of drugs with a known risk of TdP.(13) As a consequence, medication surveillance alerts for the interaction between ciprofloxacin and QT prolonging drugs are incorporated in many healthcare systems. If ciprofloxacin does not prolong the QT interval, the alerts and the precautionary measures taken to reduce the risk of $\mathrm{TdP}$ would be redundant. These measures vary from taking additional ECGs and 
monitoring the QT interval during therapy, to switching to alternative antimicrobial agents and possibly withholding the treatment of first-choice.

\section{CONCLUSION}

To conclude, we did not find any QT prolonging effect after intravenous administration of ciprofloxacin in ICU patients. No observational study or cohort study thus far has shown that ciprofloxacin has a QT prolonging effect or increases the risk of $\mathrm{TdP}$ or (cardiovascular) mortality. Based on our results and the data from previous clinical studies, ciprofloxacin monotherapy lacks any clinically relevant QT prolonging effect.

\section{REFERENCES}

1. Rubinstein E, Camm J. Cardiotoxicity of fluoroquinolones. $J$ Antimicrob Chemother, 2002;49:593-596.

2. Roden DM. Drug-induced prolongation of the QT interval. $N$ Engl J Med, 2004;350:1013-1022. doi: 10.1056/NEJMra032426

3. Ball P. Quinolone-induced QT interval prolongation: a not-so-unexpected class effect. $J$ Antimicrob Chemother, 2000;45:557-559.

4. Mehrzad R, Barza M. Weighing the adverse cardiac effects of fluoroquinolones: A risk perspective. $J$ Clin Pharmacol, 2015;55:1198-1206. doi: 10.1002/jcph.553.

5. Haring B, Bauer W. Ciprofloxacin and the risk for cardiac arrhythmias: culprit delicti or watching bystander? Acta Cardiol. 2012;67:351-354. doi: 10.2143/AC.67.3.2160727

6. Daya SK, Gowda RM, Khan IA. Ciprofloxacin- and hypocalcemia-induced torsade de pointes triggered by hemodialysis. Am J Ther, 2004;11:77-79.

7. Makaryus AN, Byrns K, Makaryus MN, Natarajan U, Singer C, Goldner B. Effect of ciprofloxacin and levofloxacin on the QT interval: is this a significant "clinical" event? South Med J, 2006;99:52-56.

8. Noel GJ, Natarajan J, Chien S, Hunt TL, Goodman DB, Abels R. Effects of three fluoroquinolones on
QT interval in healthy adults after single doses. Clin Pharmacol Ther, 2003;73:292-303.

9. Tsikouris JP, Peeters MJ, Cox CD, Meyerrose GE, Seifert CF. Effects of three fluoroquinolones on QT analysis after standard treatment courses. Ann Noninvasive Electrocardiol, 2006;11:52-56. doi: 10.1111/j.1542-474X.2006.00082.x

10. Ray WA, Murray KT, Hall K, Arbogast PG, Stein $\mathrm{CM}$. Azithromycin and the risk of cardiovascular death. $N$ Engl J Med, 2012;366:1881-1890. doi: 10.1056/NEJMoa1003833

11. Chou HW, Wang JL, Chang CH, Lai CL, Lai MS, Chan KA. Risks of cardiac arrhythmia and mortality among patients using new-generation macrolides, fluoroquinolones, and beta-lactam/beta-lactamase inhibitors: a Taiwanese nationwide study. Clin Infect Dis, 2015;60:566-577. doi: 10.1093/cid/ciu914

12. Inghammar M, Svanström $H$, Melbye M, Pasternak B, Hviid A. Oral fluoroquinolone use and serious arrhythmia: bi-national cohort study. $B M J$, 2016;352:i843. doi: 10.1136/bmj.i843

13. CredibleMeds ${ }^{\circledR}$. Center of Education and Research in Therapeutics of the University of Arizona (AzCERT). Available at: https://www.crediblemeds.org/ (accessed 21.11.2016)

14. Duprey MS, Al-Qadheeb N, Roberts R, Skrobik Y, Schumaker G, Devlin JW. The use of low-dose IV haloperidol is not associated with QTc prolongation: post hoc analysis of a randomized, placebocontrolled trial. Intensive Care Med, 2016;42:18181819. doi: 10.1007/s00134-016-4512-3

15. Bazett HC. An analysis of the time-relations of the electrocardiograms. Heart, 1920;7:353-370.

16. Fridericia LS. Die systolendauer im elektrokardiogramm bei normalen menschen und bei herzkranken. Acta Med Scand. 1920;53:469-486.

17. Vandenberk B, Vandael E, Robyns T, Vandenberghe J, Garweg C, Foulon V, Ector J, Willems R. Which QT Correction Formulae to Use for QT Monitoring? $J$ Am Heart Assoc, 2016 Jun 17;5(6). pii: e003264. doi: 10.1161/JAHA.116.003264

18. Medicines Evaluation Board (CBG-MEB). Summary of Product Characteristics Ciprofloxacin. Available at: https://db.cbg-meb.nl/mri/spc/nlh0302-001-002-003.pdf (accessed 2.10.2017) 